\title{
ON AN ABSOLUTE CONSTANT PERTAINING TO CAUCHY'S “PRINCIPAL MODULI" IN BOUNDED POWER SERIES
}

\author{
AUREL WINTNER
}

1. Let $f(z)$ be a function which is regular within the unit circle and satisfies the inequality

$$
|f(z)|<1 \text { for }|z|<1 .
$$

For $|z|<1$, let another regular function, $g(z)$, be defined by placing

$$
g(z)=\sum_{0}^{\infty}\left|c_{n}\right| z^{n} \quad \text { if } \quad f(z)=\sum_{0}^{\infty} c_{n} z^{n} .
$$

It follows from (1) by Parseval's relation (first applied, in this context, by Gutzmer; cf. [2, p. 8]) that

$$
\sum_{0}^{\infty}\left|c_{n}\right|^{2}<1, \text { hence }\left|c_{n}\right|<1,
$$

and it is also known that

$$
\left|c_{n}\right|<2\left(1-\left|c_{0}\right|\right) \text { if } n>0
$$

(this fact goes back to Carathéodory, and is connected with the Carathéodory-Toeplitz criterion for functions which are harmonic and positive in the unit circle; for a simple direct proof of (4), due to Bohr, cf. $[2$, p. 28]).

It is clear from (1) that

$$
\sup _{|z|<1}|z| f(z) \mid \geqq 1
$$

(the sup can be $\infty$ ) and that, in view of the example $f(z)=z$, the lower bound 1 cannot be improved to any greater absolute constant. In what follows, the corresponding question will be considered for the case in which $f(z)$ is replaced by $g(z)$. The result will be that

$$
\sup _{|z|<1}|z / g(z)|>1 / 3
$$

Received April 12, 1956. 
holds by virtue of (1), and that $1 / 3$ is the best absolute constant here: The question arises in connection with Cauchy's "principal moduli"; cf. [1] and, for further considerations, [3].

2. It is clear from (1) and (2) that, if $f(z) \neq$ const. and $0<r<r+h<1$,

$$
0<\max _{0 \leqq|z| \leqq r}|f(z)| \leqq \max _{0 \leqq|z| \leqq r}|g(z)|=g(r)<g(r+h)<g(1),
$$

where $g(1)$ is defined to be $g(1-0)=\left|c_{0}\right|+\left|c_{1}\right|+\ldots$, hence $g(1)=\infty$ is allowed. It is also clear that there exists a finite $\min _{0 \leqq r \leqq 1} g(r) / r$, where $g(r) / r$ at $r=0$ is meant to be $\infty$ or $\left|c_{1}\right|$ according as $\left|c_{0}\right|>0$ or $\left|c_{0}\right|=0$. Obviously, there exists at least one $r_{0}=r_{0}(g)$ satisfying

$$
\min _{0 \leqq r \leqq 1} g(r) / r=g\left(r_{0}\right) / r_{0},
$$

where $0 \leqq r_{0} \leqq 1$. It will be seen below that this

$$
r_{0} \text { is unique unless } g(r) / r=\text { const. or } g(0)=0 \text {. }
$$

It will be concluded from (4) that

$$
\min _{0 \leqq r \leqq 1} g(r) / r<3
$$

holds by virtue of (1) alone, and (6) will be convenient when proving that the 3 of (7) cannot be replaced by any smaller absolute constant. In the latter regard, it will be essential to use functions for which the $r_{0}=r_{0}(g)$ occurring in (5) is within the open interval

$$
0<r_{0}<1 \text {. }
$$

For, if (8) does not hold, then

and

$$
\min _{0 \leqq r \leqq 1} g(r) / r<\left(3+\frac{1}{2}\right)^{\frac{1}{2}}(<3) \quad \text { if } \quad r_{0}=1
$$

$$
\min _{0 \leqq r \leqq 1} g(r) / r<1 \quad \text { if } \quad r_{0}=0 .
$$

3. Consider first the case (8). In this case, it follows from (5) that the derivative of $g(r) / r$ must vanish at $r=r_{0}$. This means that

$$
r_{0} g^{\prime}\left(r_{0}\right)=g\left(r_{0}\right),
$$

where $g^{\prime}=d g / d r$. But (2) shows that, if $r<1$,

$$
r g^{\prime}(r)-g(r)=-\left|c_{0}\right|+\sum_{2}^{\infty}(n-1)\left|c_{n}\right| r^{n},
$$


and, except when $c_{n}=0$ for every $n>1$, the function (12) of $r$ is strictly increasing. Hence, if the trivial case $g(r)=\left|c_{0}\right|+\left|c_{1}\right| r$ is excluded, then the equation (11) cannot have more than one root on the interval (8). This proves (6), and more, for the case (8). If (8) does not hold, that is, if either $r_{0}=0$ or $r_{0}=1$, then (6) is obvious from (2).

It also follows that, if $r_{0}=1$, then the function (12) is negative for every positive $r<1$ (except in the trivial cases which have been excluded). Hence

$$
\sum_{2}^{\infty}(n-1)\left|c_{n}\right| \leqq\left|c_{0}\right| \quad \text { if } \quad r_{0}=1 .
$$

But (13) implies that

$$
\sum_{2}^{\infty} 2\left|c_{n}\right| \leqq \sum_{2}^{\infty} n\left|c_{n}\right| \leqq\left|c_{0}\right|+\left|c_{2}\right|
$$

Hence $g(1)=\sum_{0}^{\infty}\left|c_{n}\right|<\infty$ and

$$
\begin{gathered}
2 g(1)<\left|c_{0}\right|+\left|c_{2}\right|+2\left(\left|c_{0}\right|+\left|c_{1}\right|\right) \\
=3\left|c_{0}\right|+2\left|c_{1}\right|+\left|c_{2}\right| \leqq\left(3^{2}+2^{2}+1^{2}\right)^{\frac{1}{2}}\left(\left|c_{0}\right|^{2}+\left|c_{1}\right|^{2}+\left|c_{2}\right|^{2}\right)^{\frac{1}{2}},
\end{gathered}
$$

and so, by (3),

$$
g(1)<\frac{1}{2}\left(3^{2}+2^{2}+1^{2}\right)^{\frac{1}{2}}=\left(3+\frac{1}{2}\right)^{\frac{1}{2}} .
$$

In view of (5), this proves (9), where $r_{0}=1$. If $r_{0}=0$, then $c_{0}=0$ in (2), hence the min on the left of (5) is $\left|c_{1}\right|$, and so (10) follows from the case $n=1$ of (3).

4. In order to prove (7), it is sufficient to ascertain that

$$
g(1 / 3)<1,
$$

since $g(r) / r$ becomes $3 g(1 / 3)$ at $r=1 / 3$. But (14) is a well-known result of Bohr [2, pp. 28-29], which follows directly from (2) and (4), since

$$
g(1 / 3)<\left|c_{0}\right|+2\left(1-\left|c_{0}\right|\right) \sum_{1}^{\infty} 3^{-n}=1 .
$$

By considering the linear functions

$$
f_{a}(z)=(z-a) /(1-a z), \quad 0<a<1,
$$

(functions for which (1) is clearly satisfied), and letting $a \rightarrow 1$, Bohr also observed that the $1 / 3$ of (14) cannot be improved to any greater absolute constant. But this fact fails to imply that the 3 of (7) cannot be improved to any smaller absolute constant. This can however be concluded as follows: 
First, from (15),

$$
f_{a}(z)=-a+\left(a^{-1}-a\right) \sum_{1}^{\infty} a^{n} z^{n},
$$

where $0<a<1$. Hence, from (2),

$$
g_{a}(r)=a+\left(a^{-1}-a\right) \sum_{1}^{\infty} a^{n} r^{n} .
$$

The last three formulae imply that

$$
g_{a}(r)=2 a+(r-a) /(1-a r), \quad 0 \leqq r<1,
$$

(and (16) shows that $g_{a}(1 / 3) \rightarrow 1$ as $a \rightarrow 1$, which is Bohr's result). If (16) is inserted in (11), where $g=g_{a}$, there results for $r_{0}=r_{0}\left(g_{a}\right)$ a quadratic equation. It is readily found that, if $a(<1)$ is close enough to 1 (in fact, if $\left.a>2 / 5^{\frac{1}{2}}\right)$, then one of the roots $r_{0}$ of that equation exceeds 1 , while the other root is within the range (8) and is given by

$$
r_{0}=r_{0}\left(g_{a}\right)=\frac{a-\left(5 a^{2}-4\right)^{\frac{1}{2}}}{2\left(1-a^{2}\right)} \quad\left(4<5 a^{2}<5\right) .
$$

It follows from (17) that

$$
r_{0}=1+o(1-a)
$$

as $a \rightarrow 1$. Since this implies that

$$
\left(r_{0}-a\right) /\left(1-a r_{0}\right) \rightarrow 1 \text { as } a \rightarrow 1,
$$

it is seen from (16) that

$$
\lim _{a \rightarrow 1} g_{a}\left(r_{0}\right) / r_{0}=\lim _{a \rightarrow 1} g_{a}\left(r_{0}\right)=\lim _{a \rightarrow 1}(2 a+1)=3 .
$$

In view of (5), this proves that the 3 is the best absolute constant in (7).

5. If only (1) is assumed but the limiting cases $r_{0}=1$ and $r_{0}=0$ (cases in which (7) can be improved to (9) and (10) respectively) are disregarded, then (11) defines a unique $r_{0}$ satisfying (8). But (11) and (8) show that (5) can be written in the form

$$
\min _{0<r<1} g(r) / r=g^{\prime}\left(r_{0}\right)
$$

(where $g^{\prime}=d g / d r$ ). Consequently, the inequality (7) and its final nature can be formulated as follows:

If $f(z)$ is subject to only (1) but the limiting cases, $r_{0}=0$ and $r_{0}=1$, of (8) are excluded in (5), then

$$
g^{\prime}\left(r_{0}\right)<3
$$

and the 3 of (19) cannot be improved to any smaller absolute constant. 
Actually, (19) holds in the limiting cases of (8) also. In fact, if $r_{0}=0$, then $\left.g^{\prime}\left(r_{0}\right)=\left|c_{1}\right|\right)$, by (2), and so the case $n=1$ of (3) shows that (19) is satisfied (since $3>1$ ). In the remaining case, $r_{0}=1$. Then (2) shows that, formally,

by (13). Hence

$$
g^{\prime}\left(r_{0}\right)=g^{\prime}(1)=\sum_{1}^{\infty} n\left|c_{n}\right| \leqq \sum_{0}^{\infty}\left|c_{n}\right|-\left|c_{1}\right|,
$$

$$
g^{\prime}(1) \leqq \sum_{0}^{\infty}\left|c_{n}\right|=g(1) .
$$

It follows therefore from (9) that (19) holds also when $r_{0}=1$.

\section{REFERENCES}

1. A. Cauchy, Euvres, sér. 1, vol. IX (1896), pp. 75-81.

2. E. Landau, Darstellung und Begründung einiger neuerer Ergebnisse der Funktionen. theorie, 1. Aufl., Berlin, 1916.

3. P. Nekrassoff, Der Modul des Maximum Maximorum einer Function $f\left(r e^{p i}\right)$ in Bezug auf $\varphi$ und die Anwendung seiner Eigenschaften auf die Reihe von Lagrange, Math. Ann. 31 (1888), 337-358.

THE JOHNS HOPKINS UNIVERSITY, BALTIMORE, MARYLAND, U.S.A. 